\title{
Significados en torno al territorio antártico chileno por parte de docentes de Historia, Geografía y Ciencias Sociales del Liceo Manuel Barros Borgoño ${ }^{1}$
}

\author{
Meanings around the chilean antarctic territory by teachers of History, \\ Geography and Social Sciences of the Liceo Manuel Barros Borgoño \\ NAdia Sofía FARÍAs CÁRdEnAs* \\ "Profesora de Historia y Geografía, titulada en Universidad de Playa Ancha (UPLA), Valparaíso. \\ Ayudante Académica, UPLA \\ $\measuredangle$ nadiafariascardenas@gmail.com
}

\begin{abstract}
RESUMEN
El artículo da cuenta de una investigación cualitativa, donde a través de un estudio de caso, se indaga en cómo un grupo de docentes de la asignatura de Historia, Geografía y Ciencias Sociales, de un Liceo público y emblemático de la comuna de Santiago, significan y defienden la idea de tricontinentalidad a partir del territorio antártico chileno, temática ausente en los planes y programas de la asignatura. Se plantea la existencia de una carencia en los planes y programas del Ministerio de Educación de Chile, respecto de la temática antártica y la soberanía que Chile ejerce en el continente blanco. Finalmente se expone una propuesta de intervención curricular para la inclusión de la temática en la asignatura, sus fundamentos y proyecciones en las nuevas Bases Curriculares en el marco de la Reforma Educacional en curso.
\end{abstract}

PALABRAS CLAVE: Antártica, educación, currículum, significados.

\begin{abstract}
The article gives an account of a qualitative investigation, where through a case study, it is investigated in how a group of teachers of the subject of History, Geography and Social Sciences, of a public and emblematic Liceo of the commune of Santiago (Chile), signify and defend the idea of tricontinentality from the Chilean Antarctic territory, subject absent in the plans and programs of the subject. It is suggested that there is a lack in the plans and

1 Artículo pertenece a proyecto de investigación/ Tesis para optar al grado de Magíster en Educación mención Currículum y Comunidad Educativa, Universidad de Chile. 2018.
\end{abstract}


programs of the Ministry of Education of Chile, with respect to the Antarctic issue and the sovereignty that Chile exercises in the white continent. Finally, a curricular intervention proposal is presented for the inclusion of the subject in the subject, its foundations and projections in the new Curricular Bases within the framework of the current Educational Reform.

KEY WORDS: Antarctic, Education, Curriculum, Perceptions.

\section{INTRODUCCIÓN}

Desde que Chile fijó los límites del territorio chileno antártico durante el gobierno de Pedro Aguirre Cerda el 6 de noviembre de 1940, lo que fue publicado el 21 de junio de 1955 en el período de Carlos Ibáñez del Campo, cuatro años después el país suscribiría al Tratado Antártico en donde se estableció que: "Las partes se comprometen a la protección global del medio ambiente antártico y los ecosistemas dependientes y asociados (...) los que designan a la Antártida como reserva natural, consagrada a la paz y a la ciencia (Tratado Antártico, 1959).

Desde aquel entonces el país ha defendido su carácter de tricontinental, es decir, que posee soberanía en tres continentes: América, Oceanía y Antártica, lo que culminaría con el proceso de conformación del territorio nacional comenzado tras su Independencia y posterior consolidación de la República a lo largo del siglo XIX.

Sin embargo, a nivel planes y/o programas de los organismos vinculados a educación en Chile durante el siglo XX, el territorio chileno antártico ha sido por lo general abandonado desde la perspectiva de la soberanía nacional y solo considerado desde los aportes en las áreas científicas, vale decir, referido a estudios de flora, fauna y relieves, dejando a un lado la relevancia que dicho territorio tiene en relación a nuestra soberanía. De este modo se encuentra escasa por no decir nula referencia a estos actos y en especial a la colonización del territorio mediante el emplazamiento de Villa Las Estrellas en la década de los 80 .

Frente a esto, surge la interrogante ¿cómo se reafirma o se defiende el carácter tricontinental del país si no es considerada desde los planes y programas de nuestro currículum, instancia necesaria para promover la importancia que tiene el que Chile ejerza presencia en el continente antártico? Esto, tanto para defender nuestra identidad territorial y que no solo se convierta en una enunciación carente de fundamento concreto, como también el valorar nuestra presencia en el último continente del mundo que no ha sido intervenido por el ser humano, destinado únicamente para fines de investigación “(...) reconociendo además las oportunidades únicas que ofrece la Antártida para la observación científica y la investigación de procesos de importancia global y regional.” (Tratado Antártico, 1959) y de cierto modo, a la preservación de la paz entre los países soberanos. 
Cabe destacar el valor que posee la Antártica como único territorio del mundo para estos fines, alejado de las intervenciones humanas, muchas veces nocivas, para nuestro ecosistema. Por otro lado, la Antártica es un territorio de relevancia global y que en base a las relaciones internacionales que desde él emanan, a la cooperación y la investigación, es necesario incorporar la temática a nuestra educación desde el proceso de continuar educando para la globalización.

Por consiguiente, el problema de investigación que se plantea, apunta a la existencia de una carencia de la temática antártica y la soberanía que Chile ejerce en ella en los planes y programas de Historia, Geografía y Ciencias Sociales, por ello, cabe cuestionar el papel que juega la educación formal al momento de crear y defender la identidad tricontinental del país, considerando que es en la escuela donde se produce el primer acercamiento al contenido concreto sobre la temática. O dicho de otra manera; ¿Cómo se defiende la tricontinentalidad del país y la soberanía chilena en la Antártica, desde la educación formal en relación a la perspectiva de docentes de Historia, Ciencias Sociales y Geografía?

Desde el punto de vista teórico, centramos nuestro análisis a partir del concepto de currículum prescriptivo, el cual está vinculado al conjunto de contenidos fijados en un marco de lo ideal, lo que sin duda es estipulado de acuerdo a dimensiones ideológicas, éticas, entre otras (Glatthom, 1994). El currículum prescriptivo, en este sentido, debe ser capaz de responder a la mayor parte de las transformaciones curriculares de la región "(...) ya que la educación escolar tiene la responsabilidad de contribuir a contrarrestar, revertir y prevenir los grandes problemas de la sociedad moderna, tales como la pobreza, la violencia, la destrucción del medio ambiente" (Ferrer, Valverde \& Esquivel, 1999. p. 4), situación que observamos de manera concreta en la necesidad de vincular la temática de la Antártica al currículum en concordancia con esta responsabilidad.

Ahora bien, son primordiales los significados que las(os) docentes otorgan a la necesidad de introducir la temática expuesta en la red de contenidos prescrita y en el marco de lo ideal en educación. Una posible propuesta de intervención curricular contempla la construcción de ella de manera intersubjetiva desde el currículum, en especial si consideramos que es una oportunidad que no existe para los docentes de aula. En este sentido, teorías conocidas por la sociología como el interaccionismo simbólico da especial papel a las interacciones humanas y la construcción social de la realidad. Tales postulados son realizados también por autores como Berger y Luckmann (1986) los que apuntan a la construcción de un mundo subjetivo entre los individuos participantes de un mundo común, en ese contexto, de una red de contenidos ideales que pretenden incorporar problemas de la sociedad contemporánea en ellos.

Desde esa mirada, el currículum corresponde en nuestra educación a un mundo objetivo, en el cual surgen procesos de internalización: la aprehensión o interpretación inmediata de este, se vuelve un acontecimiento subjetivo en cuanto expresa significado para los sujetos partícipes de esta investigación, es decir, cuando éste se construye desde el marco de referencia de los sujetos (Berger \& Luckmann, 1986). Por ello es de especial interés que 
si existe la pertinencia de crear una intervención curricular en temas antárticos, ésta sea desde la perspectiva subjetiva de los docentes a fin de que se vuelva significativa para su aplicación. Se trata de re significar el contenido curricular desde las perspectivas subjetivas de un currículo objetivo que hoy se imparte ajeno a los significados de quienes lo aplican.

\section{Aspectos Metodológicos}

El trabajo se inscribe al alero del paradigma interpretativo, cuyo “(...) objeto es el desarrollo de conceptos que ayuden a comprender los fenómenos sociales en medios naturales dando la importancia necesaria a las intenciones, experiencias y opiniones de todos los participantes" (Martínez, 2013. p. 5), lo que se tradujo en el análisis de los significados que los sujetos, en este caso, docentes de la asignatura den al territorio antártico chileno y su vinculación al currículum nacional como conocedores de éste.

En términos metodológicos, la presente investigación corresponde a un estudio de caso, puesto que los sujetos partícipes provienen de un mismo establecimiento y aplican el mismo currículum en sus prácticas cotidianas.

En este sentido, los criterios que se usaron para seleccionar a los participantes de esta investigación fueron, en correlación:

- Docentes titulados de Historia y Geografía y/o Ciencias Sociales

- Docentes de Historia y Geografía y/o Ciencias Sociales que realicen clases de la disciplina en niveles de primero a cuarto medio en el Liceo Municipal Liceo Manuel Barros Borgoño

- Docentes que conozcan y apliquen el Currículum nacional en sus prácticas docentes

Además, dada las condiciones del liceo en términos de rotativa en cargos administrativos, se incluyó, en una segunda entrevista grupal a docentes de la asignatura que a la fecha de la realización de esta investigación no se encontraban realizando clases en aula, pero el cese de sus funciones en la misma comenzó hace menos de 3 años, esto porque en el marco de las reformulaciones curriculares que se están llevando a cabo desde el Ministerio de Educación, estos docentes si aplicaron el currículum vigente a lo largo de su experiencia laboral y pueden otorgar significados desde aquella misma experiencia.

\section{Muestra}

El tipo de muestra utilizado en esta investigación, es no probabilístico, es decir, la elección de los participantes no fue elegida al azar, sino que se buscó encontrar representatividad para la problemática expuesta, es decir, la comprensión de significados en torno al territorio antártico chileno en el currículum de Historia, Geografía y Ciencias Sociales. 
Bajo esta mirada de muestreo no probabilístico, la muestra corresponde a una de tipo intencional o de conveniencia, que en palabras de Canales, está “(...) caracterizada por la intencionalidad consciente y expresa de crear vínculos entre lo conocido y lo desconocido, entre la teoría y la práctica, entre el yo y los otros, entre los discursos y la acción que transforma la realidad" (2006. p. 351).

Los docentes partícipes de esta investigación pertenecen al Liceo Manuel Barros Borgoño (A-10), establecimiento municipal de la comuna de Santiago (Región Metropolitana, Chile). El establecimiento mencionado fue seleccionado debido a que al ser municipal, implementa y aplica el currículum nacional bajo los lineamientos que establecen las políticas nacionales de educación.

En este sentido, los criterios que se usaron para seleccionar a los participantes de esta investigación fueron, en correlación:

- Docentes titulados de Historia y Geografía y/o Ciencias Sociales

- Docentes de Historia y Geografía y/o Ciencias Sociales que realicen clases de la disciplina en niveles de primero a cuarto medio en el Liceo Municipal Liceo Manuel Barros Borgoño

- Docentes que conozcan y apliquen el Currículum nacional en sus prácticas docentes

La recolección de datos se realizó a través de la técnica de grupo focal incorporando entrevista semi estructurada. La importancia de realizar un grupo focal radica en la experiencia vivida de los sujetos (Merton, 1965) en un diálogo compartido con otros, que en este caso, comparten cierta base para la creación de su percepción particular.

Por otro lado, se utilizó el grupo focal, puesto que el objetivo de esta técnica en la investigación social es "lograr una información asociada a conocimientos, actitudes, sentimientos, creencias y experiencias que no serían posibles de obtener, con suficiente profundidad, mediante otras técnicas tradicionales tales como por ejemplo la observación, la entrevista personal o la encuesta social.” (Aigneren, 2002, p. 8). En este sentido, la interacción entre los docentes entrevistados fue primordial y base para el posterior análisis de los datos recogidos, ya que aquello aportó a una mayor comprensión de los fenómenos asociados al currículum y al territorio antártico chileno a diferencia de entrevistas individuales, donde la percepción de los docentes se encontraría aislada y carente de la interacción grupal que permite un comprensión más integral de los significados.

Concretamente, se recurrió a la entrevista "enfocada o centrada", caracterizada como “(...) un intento por combinar parte de las dimensiones asociadas a la profundidad y la libertad que observan las entrevistas no estructuradas con las características de las entrevistas estructuradas." (Ruiz e Ispizúa, 1989. p. 154 en Agulló, 1998), es decir, una entrevista que esté construida con un foco pero que no descarte la posibilidad de desviarlo hacia otras dimensiones para luego volver a él. 
Por consiguiente, se propusieron las siguientes preguntas que sirvieron de entrada o de guía para la realización de las entrevistas.

1) ¿Abordan la temática del territorio antártico chileno en sus clases? De hacerlo, ¿A qué aspectos le dan más relevancia? De no hacerlo, ¿A qué atribuye el no abordaje de la temática?

2) ¿Por qué creen uds que la soberanía de nuestro país en el territorio antártico chileno, no ha sido suficientemente abordada en nuestros planes y programas de Historia y Geografía en educación?

3) ¿De qué manera creen uds que afecta en la ciudadanía el hecho de que la temática no esté incorporada en nuestros planes y programas, en relación a nuestra identidad nacional?

4) ¿En qué nivel, unidad y/o contenido creen uds que se aborda mejor la soberanía en el territorio antártico chileno? ...

Se dejó una quinta pregunta con puntos suspensivos (...) para dar la libertad al grupo focal de crear su propia línea de discusión, es decir, estas preguntas corresponderían al eje o foco de la entrevista, pero al ser esta una entrevista que pretende ser "enfocada o centrada", las preguntas pudieron también dar origen a otras, con el fin de indagar en otras dimensiones del contenido y significados en los entrevistados.

Tabla 1. Niveles y contenido curricular propuestos por el entrevistador (de acuerdo al currículum conocido y aplicado por los sujetos entrevistados) ${ }^{2}$.

\begin{tabular}{|l|l|l|}
\hline \multicolumn{1}{|c|}{ Nivel } & \multicolumn{1}{|c|}{ Unidad / Contenido } & \multicolumn{1}{|c|}{$\begin{array}{c}\text { Horas } \\
\text { Pedagógicas }\end{array}$} \\
\hline Segundo medio & $\begin{array}{l}\text { Unidad 3 "Conformación del territorio chileno y sus } \\
\text { dinámicas geográficas". }\end{array}$ & 34 Horas \\
\hline Tercero medio & $\begin{array}{l}\text { Unidad 1 "Transformación del rol del Estado y } \\
\text { modernización de la sociedad en la primera mitad del } \\
\text { siglo XX". }\end{array}$ & 42 Horas \\
\cline { 2 - 4 } & $\begin{array}{l}\text { Unidad 3 "El quiebre de la democracia y la dictadura } \\
\text { militar". }\end{array}$ & 36 Horas \\
\hline Cuarto medio & $\begin{array}{l}\text { Unidad 3 "Los desafios de insertarse en un mundo } \\
\text { globalizado: Chile y sus regiones frente a la economía } \\
\text { y los problemas globales". }\end{array}$ & 30 Horas \\
\hline
\end{tabular}

Extraído de Planes y Programas, Historia, Geografía y Ciencias Sociales. Ajustes 2009 para Segundo, Tercero y Cuarto Medio. Ministerio de Educación, Chile. Previo a los ajustes curriculares comenzados con la última reforma educacional (2014) y que se comenzarían a impartir durante el año 2018. Disponibles en: www. curriculumnacional.cl 
Además, se propusieron los niveles y unidades posibles de intervenir curricularmente, en relación al currículum conocido por los sujetos entrevistados:

Lo anterior se plantea a modo de propuesta, atendiendo a que desde la perspectiva curricular, presenta congruencia con el abordaje de la soberanía en el territorio antártico chileno y la defensa de la tricontinentalidad de Chile y además es coherente con el tipo de entrevista, "enfocada o centrada” que aquí se propone. Sin embargo, se dejó a elección del grupo de docentes entrevistados, tomar la decisión de si es necesario intervenir el currículum y en qué nivel, unidad o contenido se debiera realizar, de acuerdo a sus percepciones en torno al tema.

En relación a la cuarta pregunta, su análisis se realizó de manera diferenciada, al estar el currículum nacional en una reformulación de sus contenidos y objetivos y que para el año 2018 se comenzaron a aplicar de manera obligatoria en los establecimientos del país. Por lo tanto, los niveles y unidades propuestas por el entrevistador apuntan a analizar el currículum conocido por los entrevistados, apelando a su experiencia en él y siguiendo los objetivos de esta investigación. Sin embargo, se dió la libertad para referirse tanto a uno como a otro en la medida en que se encuentren en la esfera de conocimiento y aprehensión de la experiencia de cada participante.

Concerniente a la credibilidad, se recurrirá al criterio de Triangulación, el que en palabras de Elliot, Sanger, Wood, Hull \& Barret (1986) señala que al comparar diversas apreciaciones de un mismo fenómeno, éste deberá observarse desde donde se difiere, si se está de acuerdo o en desacuerdo, es en estos puntos de encuentro o desencuentro donde puede validarse teóricamente la investigación.

Dentro de los tipos de Triangulación que pueden aplicarse a los datos recogidos en el instrumento propuesto, se utilizará la llamada Triangulación del Evaluador, la que en palabras de Casanova (1998) "Supone que son varios los profesores que intervendrán en el proceso evaluador, aplicando las técnicas acordadas para la obtención de datos y luego contrastando los mismos y comprobando su coincidencia” (Casanova, 1998, p. 8), entendiendo que participarán otros expertos en el trato de los datos, los que analizarán las técnicas y los instrumentos de recolección de los mismos que se han expuesto como parte de este proyecto de investigación.

Fue necesario que la validación se realizará analizando los aspectos antes mencionados por expertos en tres áreas principales; la que respecta a la temática Antártica y en particular al territorio antártico chileno y corresponderá a expertos vinculados al Centro de Estudios Hemisféricos y Polares (CEHP), organización que busca "Estudiar, promover, publicar y difundir desde perspectivas interdisciplinarias o especializadas las realidades de las naciones que componen el Hemisferio Occidental, las Regiones Polares y su gravitación e impactos internacionales ${ }^{3}$ (Misión, CEHP), única instancia académica de la zona central que ha realizado intervenciones de diversas índoles referidas a la Antártica en el medio escolar.

Página institucional: Centro de Estudios Hemisféricos y Polares. http://www.hemisfericosypolares.cl/ 
Por otro lado, se recurrió a expertos en el área de investigación cualitativa con el fin de validar el instrumento y las técnicas que se proponen, además de expertos en educación que evaluaron los lineamientos y objetivos curriculares que se plantean en este proyecto.

Sin embargo, al momento de triangular los datos recogidos, y al estar basados en las percepciones otorgadas por los entrevistados, otorgaron mayor credibilidad a la investigación al estar centradas en los significados que otorguen las personas entrevistadas, dada sus percepciones, puntos de vistas, reflexiones y experiencias sometidas a análisis, buscando puntos de encuentros y desencuentros, a modo de construir una idea en común del lugar y la importancia que debiera tener nuestra antártica en los planes y programas que enseñamos, al menos en Historia y Geografía, a lo largo de todo Chile, un país tricontinental que deja una gran parte del territorio nacional ausente del imaginario y la identidad de nuestro país.

\section{ANTECEDENTES REFERIDOS A LA VINCULACIÓN EDUCACIÓN Y ANTÁRTICA}

En lo concerniente a las investigaciones que se han realizado en torno a la Antártica y Educación, podemos mencionar interesantes artículos que vinculan ambas áreas y que por lo general, se desarrollan fuera de la esfera nacional. En este sentido y a nivel continental destaca el artículo "La Antártida, lugar de investigación y docencia” de Antiveros, Alonso y Vilchez (2011), publicación realizada en la revista electrónica Actualidades Investigativas de Educación de la Universidad de Costa Rica, donde se establece la importancia de estudiar ciencia conectando los dominios académico, científico y cotidiano y en este caso, aplicándolo a un trabajo cuyo escenario fue la Antártica y en el cual se pudieron conectar "los dominios científico y académico de un modo poco habitual en la docencia actual, quedando ambos fundidos en, y con, nuestra vida cotidiana, (...) mejoraríamos la docencia de las asignaturas científicas (p. 23), haciéndola más cercana al alumnado.

A nivel latinoamericano, es Argentina donde se ha escrito en mayor medida en torno a la educación y la Antártica, entre estas investigaciones señalaremos el artículo de Amelia García, “Textos escolares: Las Malvinas y la Antártida para la "Nueva Argentina” de Perón” (2009), quien investigó sobre el imaginario antártico en los textos escolares argentinos durante 1946 y 1955, periodo en que el país vecino suscribió al Tratado Antártico, probando la presencia en tales documentos de “(...) la defensa de la soberanía nacional en el lejano sur y la formación de la conciencia antártica como dos ideas ejes que en líneas generales estructuran la información en los textos escolares que circularon durante el primer período peronista" (p. 1040).

Artículo similar es el titulado "Mi ciudad y la Antártida llegan a la escuela, educación ambiental y las TIC en la formación docente y en el aula” (2015) de María Andechaga, quien nos habla de cómo con ayuda desde la Antártida, mediante diferentes redes sociales se diseñó un Power Point explicativo de la base que fue utilizado para iniciar las clases y generar la necesidad en los estudiantes de buscar más información “(...) Se comparaba la ciudad con 
la Antártida analizando los materiales, el agua, la energía y los seres vivos (p. 7). Importante innovación en torno a tecnologías educativas y la Antártica.

Otro documento que se puede mencionar en torno al tema a tratar, corresponde a un tipo de texto que podríamos llamar manual o guía sobre la Antártica, y que se titula "Antártida Educa". Dicho documento es de autoría española, pero creado en Buenos Aires a través del Centro Cultural de España en Buenos Aires ${ }^{4}$ y quien declara defender desde el marco de la multilateralidad, “(...) la necesidad de garantizar la provisión de los llamados bienes públicos globales, como la paz, la libertad o la calidad medio ambiental” (Pajín, 2005 en Poves, 2005), donde por esencia, el territorio antártico se inscribe dentro de tales bienes públicos al ser un espacio destinado a la paz y el cuidado del medio ambiente.

A nivel chileno, si bien es cierto que no se han realizado muchas investigaciones en torno a Antártica y Educación, si es necesario mencionar la postura y los lineamientos que tiene el país en torno al tema, dada la importancia y cercanía geográfica que tiene con el continente antártico, como también la presencia como país consultivo en el Tratado Antártico desde 1959. Tal como señalan Gorostegui y Waghorn ${ }^{5}$ en un completo estudio que repasa la historia diplomática y científica de Chile en el continente blanco; "Siendo Chile continental un país con proyecciones naturales hacia el Continente Antártico y firmatario original del Tratado Antártico el tema antártico ocupa un lugar relevante en la agenda política del Estado desde hace ya más de medio siglo." (Gorostegui \& Waghorn, 2012. p. 13), destacando desde la perspectiva jurídica e institucional, la importancia del territorio en materia de políticas públicas y como parte de la gran proyección natural de la soberanía en continente sudamericano.

Los artículos estudiados en Chile fueron extraídos del repositorio de la Universidad de Magallanes, se encuentran en formato digital y corresponden a una tesis de pregrado para optar al título de Licenciado en Educación de la Universidad de Magallanes y cuatro monografías creadas en el marco del Diplomado en Estudios Antárticos a cargo del Centro de Investigación Antártica de la misma Universidad desde el año 2013 y que se mantiene a la fecha.

En relación con las monografías encontradas, se encuentra "Chile y el reclamo de la soberanía sobre el territorio antártico.” de Carlos Palacios Vera, escrita en el 2016, y que realiza una descripción histórica de las reclamaciones de Chile en la Antártica, las que subdivide en tres ejes fundamentales; Antecedentes Históricos, Proximidad y Continuidad Geográfica y Actividad y Ocupación Efectiva de Chile en el territorio. Agrega además, que los esfuerzos que Chile ha realizado por reforzar su posición y soberanía en la Antártica, deben ser coherente con el Plan Estratégico de Política Nacional Antártica actual a través de medidas específicas y concretas, constantes y sostenidas en el tiempo.

Creado por CCEBA; Centro Cultural de España en Buenos Aires. Página institucional http://www.cceba.org.ar/ José Gorostegui, Rodrigo Waghorn. “Chile en la Antártica; nuevos desafíos y perspectivas”. Estudio enmarcado en proyecto de tesis para optar al grado de Magíster en Política Exterior. USACH. Abril, 2012. 
Otra monografía de este tipo perteneciente el Diplomado de Asuntos Antárticos, corresponde a "Aproximaciones sobre las invasiones biológicas en la Antártica para estudiantes de segundo ciclo básico ¿cómo conservar el continente blanco?” de Javiera Mayanz Labra. Escrita en 2014, intenta vincular dos áreas muy similares a la investigación que acá se está desarrollando; Antártica, vista desde la mirada de las Ciencias Naturales y Educación, particularmente en el segundo ciclo básico.

Similar a lo anterior, destaca un trabajo titulado "Relación histórica entre el continente blanco y nuestro país y el rol de la asignatura de Historia en la enseñanza de contenidos Antárticos" escrita en agosto del 2016, por Adriana Panichini Miranda, el que se podría decir, es el que más se acerca a los objetivos de estudio de esta investigación.

La última monografía que se inscribe en este mismo programa académico y que utilizaremos como parte de esta sección, se titula "Monografía Nivelación de Competencias y Temas Antárticos.” de la profesora Marlene Mónica Alvarado Arteaga. Escrita en el 2013, año en que inició el diplomado de Asuntos Antárticos, pretende, en una abierta crítica al paradigma conductista y la importancia que tiene el guiar y estimular que los estudiantes de educación superior puedan formarse a descripción de estas técnicas, enfatizando en cómo cada una de ellas podría contribuir a lograr mayores competencias en los estudiantes de educación superior.

Además de estas monografías descritas, debemos mencionar los aportes que realiza el proyecto de tesis para optar al grado de Licenciatura en Educación titulado "Propuesta de inclusión de contenidos histórico- culturales al Programa de Primer Año Medio de los establecimientos educacionales de la región de Magallanes y Antártica Chilena, asociados a la promoción de la Identidad Regional” de las autoras Marlove Gaete, Viviana González y Flor Leiva. Tal tesis fue evaluada por la Universidad de Magallanes, Punta Arenas y defendida en el año 2007.

Sin embargo, con las actualizaciones realizadas en el 2009 al currículum nacional, los programas de Historia, Geografía y Ciencias Sociales se vieron fuertemente rediseñados, relegando los contenidos de Geografía, ampliamente tratados en primero medio durante el 2007 a niveles de enseñanza básica. De este modo, los contenidos de primero medio pasarían a tratar temas vinculados a los procesos históricos del siglo XX a nivel mundial, en cuyos propósitos estarían:

“El estudio de Historia, Geografía y Ciencias Sociales es relevante en la formación de los estudiantes de Chile, dado que les permite construir una imagen del mundo en que viven, situándolos en el tiempo y en el espacio, y les entrega herramientas para comprender el entorno social en que se desenvuelven. Se busca que desarrollen competencias y habilidades que les posibiliten un mejor conocimiento de cómo se ha constituido la realidad del presente, para así discernir sus opciones y trazar planes a futuro"' (MINEDUC. 2011. p. 24).

6 Programa de Estudio Primer Año Medio, Historia, Geografía y Ciencias Sociales. MINEDUC. Chile, primera edición, 2011. 
Así, los temas relacionados a la creación de una identidad regional se abandonarían para dar pie a una visión nacional de ciertos procesos, hitos y eventos que marcan nuestra historia como humanidad y en donde nuevamente la Antártica no estaría presente en los contenidos curriculares de la disciplina.

A nivel internacional, podemos mencionar en primera instancia el importante trabajo que realiza la University of Canterbury, en Nueva Zelanda, Universidad fundada en 1873 y la segunda Universidad de dicho país, además de estar situada en el lugar 214 del ranking (QS) que realiza de manera privada la compañía Quacquarelli Symonds ${ }^{8}$ a nivel mundial ${ }^{9}$. Esta Universidad posee un amplio e interesante Journal sobre Antarctic Studies ${ }^{10}$, el que recoge diversos artículos sobre Antártica en áreas vinculadas a las ciencias, es especial a la biología, tecnología y química, entre otras. Los artículos de esta plataforma de la UC, están además, validados por la base de datos bibliográficos Scopus, lo que la constituye como una herramienta digital de rigurosidad científica.

A pesar de que el enfoque de la plataforma descrita está centrado en estudios científicos, se ha encontrado información referente a Antártica y Educación en el artículo "Antarctic Future - Education Learning about Antarctica in the 21st Century. A review of selected education resources available to primary teachers", (2011) de Emma McFadyen. En él, se pretende hacer una revisión de dos documentos oficiales de Nueva Zelanda; The New Zealand Curriculum (Ministry of Education, 2007) y el Guidelines for Environmental Education in New Zealand Schools (Ministry of Education, 1999) ${ }^{11}$ para, en base a cinco competencias presentes en ellos: thinking, using language, symbols and text, managing self, relating to others and participating and contributing (McFadyen, 2011), poder desarrollar las capacidades y posibilidades de los docentes de potenciar en los estudiantes la importancia que tiene la Antártica en el futuro.

Muy similar al caso anterior, pero ya más de carácter práctico, encontramos la plataforma "Classroom Antarctic"12, creada por la Australian Antarctic Division, del Departamento de Medio Ambiente y Energía, perteneciente al Gobierno Australiano. Esta herramienta es especialmente interactiva y funciona de manera online para su uso y ejecución, en ella, se sugieren actividades para estudiantes de 3 er a 8 vo grado de primaria, en la que se incluyen objetivos, secuencia de actividades, evaluaciones, insumos audiovisuales, entre otros.

Por otro lado, la Universidad de Cambridge en Inglaterra, también posee un portal digital que contiene artículos de rigurosidad científica llamado Cambridge Core, the home of academic content ${ }^{13}$, esta plataforma fue diseñada para ayudar a los lectores a

University of Canterbury, New Zeland. http://www.canterbury.ac.nz/

Quacquarelli Symonds; http://www.qs.com

Para establecer un punto de referencia con nuestro contexto nacional, la Universidad de Canterbury se encuentra 14 lugares bajo el lugar que ocupa la Universidad de Chile en el mismo ranking.

Antarctic Studies; http://canterbury.libguides.com/anst/articles

New Zeland Curriculum; http://nzcurriculum.tki.org.nz/The-New-Zealand-Curriculum

Classroom Antarctica. https://classroom.antarctica.gov.au/

Cambridge Core. https://www.cambridge.org/core/ 
realizar búsquedas más rápidas y fáciles en amplios contenidos en investigación. El portal contiene una sección llamada “Antarctic Science” con una amplia difusión de estudios interdisciplinarios sobre gestión ambiental, biodiversidad, estructuras geográficas, oceanografía. Es además una revista que no limita la extensión de los artículos ni el cobro extra por material icónico y que publica editoriales periódicamente sobre temas que considera relevante en el estudio antártico.

Continuando con los aportes prácticos que ha hecho Inglaterra, desde el mismo gobierno inglés y desarrollada por la Royal Geographical Society en colaboración con British Antartic Survey; Natural environment research council y el Foreign \& Commonwealth Office, encontramos la plataforma “Discovering Antarctica: The world's last great wilderness"14, creada en 2016 para dar a conocer los efectos del cambio climático en la Antártica, lo que está apoyado por otras informaciones que invitan a los lectores a conocer acerca de sus océanos, atmosfera, paisajes, ecosistemas naturales, ciencia, exploraciones, turismo, desafíos y formas de gobernar el territorio. Puede comprenderse la existencia de este último aspecto y dado el contenido que este posee, en que es importante para el gobierno inglés dar a conocer la presencia del país en el continente blanco.

Estados Unidos, por su parte, también ha realizado contribuciones al conocimiento antártico a través del USAP; United States Antarctic Program ${ }^{15}$, programa que representa los intereses estadounidenses en la Antártica de acuerdo a los objetivos establecidos en el Tratado Antártico; mantener la cooperación internacional y la presencia activa e influyente de la región. Cabe mencionar en este sentido, el programa Polar Palooza que reúne periódicamente a científicos de distintas bases científicas en la Antártica en encuentros de investigación científica a modo de compartir resultados de ellos entre los países.

Volviendo a Chile, desde las esferas gubernamentales es necesario mencionar las actividades que realiza periódicamente el Instituto Antártico Chileno, creado en Santiago en 1963 para velar por el quehacer científico, como “(...) único organismo estatal responsable de coordinar, planificar y ejecutar esta acción, centralizando y desarrollando en este organismo las actividades que se ejecuten en el Territorio Chileno Antártico ${ }^{16 "}$ (INACH, 2018), fue trasladado en 2003 a Punta Arenas donde opera en la actualidad, lo que otorga a esta institución gran valor a nivel regional en el quehacer antártico.

Citando la misión institucional del INACH, encontramos:

[Cumplir con la Política Antártica Nacional, incentivando el desarrollo de la investigación científica, tecnológica y de innovación en la Antártica, siguiendo cánones internacionales, participando efectivamente en el Sistema del Tratado Antártico y foros

\footnotetext{
Discovering Antarctica. https://discoveringantarctica.org.uk/\# USAP. https://www.usap.gov/

Instituto Antártico Chileno: http://www.inach.cl/
} 
internacionales relacionados, fortaleciendo a Punta Arenas como puerta de entrada al Continente Blanco, realizando acciones y actividades de divulgación y valoración del conocimiento antártico en la comunidad nacional, y asesorando a la autoridad en materias antárticas. ${ }^{17}$ (INACH, 2018).

La importancia del INACH para los fines de esta investigación radica en las actividades de divulgación del conocimiento antártico a nivel educacional, lo que se potencia a través del Departamento de Comunicaciones y Educación. En tal departamento y además correspondiente a la labor de divulgación de la temática antártica se encuentra la más importante actividad escolar que vincula Antártica y Educación; la "Feria Antártica Escolar" (FAE). Esta instancia creada por el Instituto Antártico Chileno en alianza con Programa Explora de la Comisión Nacional de Investigación Científica y Tecnológica (CONICYT) y el Centro de Investigación Dinámica de Ecosistemas Marinos de Altas Latitudes (IDEAL) ${ }^{18}$, dependiente de la Comisión Nacional de Investigación Científica y Tecnológica, financiado por INACH y liderado por la Universidad Austral en asociación con la Universidad de Concepción y el Centro de Estudios del Cuaternario, FuegoPatagonia y Antártica (CEQUA) en Punta Arenas.

Otra institución presente en Chile y que se ha dedicado a estudiar la temática antártica corresponde al Centro de Estudios Hemisféricos y Polares ${ }^{19}$ (CEPH), institución de carácter privado y que ha desarrollado diversas investigaciones e instancias afines a la Antártica, en las que destacan publicaciones, organización de Congresos, entre otros.

El CEPH fue fundado en 2006 y está financiado por la Fundación Valle Hermoso ${ }^{20}$, institución creada dos años antes con personalidad otorgada por el Ministerio de Justicia el 14 de Abril de 2004. El objetivo de esta Fundación radica en:

[(...) conservar, proteger e incrementar el patrimonio histórico, cultural y ambiental de la nación chilena y fomentar el desarrollo social, la formación y capacitación laboral de los sectores más vulnerables de la población, considerando prioritariamente las necesidad de las mujeres y niños.] (Fundación Valle Hermoso, 2018).

A su vez, la Fundación trabaja a través del Centro de Estudios Hemisféricos y Polares donde plantea sus áreas de desarrollo en diversas temáticas, tales como: Patrimonio Cultural, Vinculación con la Comunidad y Patrimonio Antártico, donde en esta última, se apoyan “(...) proyectos e iniciativas dedicadas a fomentar la memoria antártica nacional, tales como proyectos de investigación, archivo fotográficos, entrevistas a personas con experiencia antártica, videos, etc." (Fundación Valle Hermoso, 2018).

\footnotetext{
Instituto Antártico Chuleno: http://www.inach.cl/inach/?page_id=10963 IDEAL: http://www.centroideal.cl/

Centro de Estudios Hemisféricos y Polares: http://www.fundacionvallehermoso.cl/

Fundación Valle Hermoso: http://www.fundacionvallehermoso.cl/
} 


\section{ANÁLISIS Y RESULTADOS}

De acuerdo a lo planteado en la descripción metodológica anterior, los datos sometidos a análisis en esta investigación corresponden a dos entrevistas grupales realizadas por un lado, a los profesores que realizan clases en aula conociendo y aplicando el currículum vigente a la fecha de aplicación del instrumento el 21 de noviembre del 2017 donde participaron 4 docentes y por otro, a docentes que aun desempeñando labores en otros cargos, cesaron su trabajo en aula hace menos de 3 años, lo que, como se dijo en páginas anteriores, también constituyen un aporte desde su experiencia en el currículum analizado. Esta última entrevista se llevó a cabo el 22 de diciembre del 2017 y reunió a 3 docentes, 2 de los cuales pudieron terminar la entrevista.

A los participantes de estas dos entrevistas se les reunió dentro del establecimiento donde se hizo el estudio de caso, específicamente en un espacio cerrado donde pudiera protegerse el sonido y que correspondió a la Sala de Reuniones del Liceo. Se les informó acerca de los objetivos de esta investigación y la pertinencia de realizarlo en dicho establecimiento, además se les entregó a cada uno de los participantes un consentimiento informado escrito acerca de las implicancias de su participación y la autorización mediante su firma para ser grabados (audio) y el uso de los datos que de ahí emergiesen. Una vez leído y firmado tal consentimiento, se dio paso a las entrevistas.

Para analizar las entrevistas mencionadas y considerando que "Generalmente, el análisis de datos cualitativos comporta la segmentación en elementos singulares, especialmente cuando son de tipo textual. En el conjunto global de datos pueden diferenciarse segmentos o unidades que resultan relevantes y significativas." (García, Rodríguez \& Gil, 1996), los elementos singulares fueron agrupados en categorías, las que entenderemos como:

“(...)ordenadores epistemológicos, campos de agrupación temática, supuestos implícitos en el problema y recursos analíticos como unidades significativas dan sentido a los datos y permiten reducirlos, compararlos y relacionarlos. Categorizar es poner juntas las cosas que van juntas. Es agrupar datos que comportan significados similares". (Galeano, 2004, citado en Romero, 2005, p 2)

La tarea de categorizar los datos recogidos en las entrevistas fue realizada por un programa digital especializado en el análisis de datos cualitativos llamado Nvivo 12 y que corresponde a la versión más actualizada del software. El procedimiento del programa mencionado se basó básicamente en insertar las entrevistas transcritas de los grupos focales, denominados "Focus Group 1" donde se encontraban las respuestas de los docentes que realizan clases en aula y que constituyó el Archivo 1 en el programa y como "Focus Group 2" con los docentes que no realizan clases en aula hace menos de 3 años, como Archivo 2. 
Los participantes fueron nombrados con un número que hace referencia al archivo donde se encuentra el registro de su respuesta y una letra A, B, C, D, E, F, G de acuerdo al orden en que aparecen sus respuestas en las entrevistas, esto con el fin de no individualizar las posturas sino que, al contrario, construir un todo para el análisis general de las percepciones de los docentes en torno al territorio antártico y la tricontinentalidad de nuestro país.

Tabla 2. Descripción categorías codificadas en Nvivo 12.

\begin{tabular}{|c|c|c|c|c|}
\hline $\mathbf{N}^{c}$ & Nombre & \multicolumn{2}{|c|}{ Referencias } & \multirow[t]{2}{*}{ Total } \\
\hline & & Archivo 1 & Archivo 2 & \\
\hline $\mathbf{1}$ & Historia y Conquista & 5 & -- & 5 \\
\hline 2 & Territorio y Soberanía & 6 & 2 & 8 \\
\hline 3 & Medio Ambiente y desarrollo científico & 12 & .. & 12 \\
\hline 4 & Tratado Antártico y proyecciones globales & 8 & 5 & 13 \\
\hline 5 & Utilidad Económica & 14 & .. & 14 \\
\hline 6 & Abordaje y Limitaciones del Currículum & 13 & 7 & 20 \\
\hline & Total & 58 & 14 & 72 \\
\hline
\end{tabular}

En este sentido, el análisis realizado con Nvivo 12 de los datos recogidos permitió crear 6 categorías codificadas de la siguiente manera:

El orden establecido de las categorías se hizo en relación a la cantidad de referencias contenidas en cada una, desde la que agrupaba menos referencias (Categoría 1, Historia y Conquista, con 5 referencias) hasta aquella que contiene mayor volumen de datos (Categoría 6, Abordaje y limitaciones del currículum, con 20 referencias). Este orden se fundamenta en una clasificación inductiva (Rodriguez et al. 1999.), en el cual la categoría con menos referencias nos permitirá avanzar en un estudio desde temas puntuales o específicos, donde el análisis pudiera verse más restringido a tales particularidades, hasta la que contenga mayor cantidad de aristas posibles de analizar y ampliar.

\section{CONCLUSIONES}

En función de la información recogida y del análisis llevado a cabo, se han podido extraer tres puntos o ejes esenciales que permien dar cuenta de lo planteado en la problemática que planteamos. Primeramente, el eje vinculado a las dimensiones objetivas - subjetivas de la acción pedagógica de los docentes en relación a la temática antártica y las tensiones en que deriva esto con la prescripción del Currículum vigente. En segundo lugar, un análisis de las percepciones de los sujetos en torno a cómo se percibe el Currículum nacional, su relación con el territorio y las necesidades a las que hoy responde en sus dinámicas, herramientas y 
orientaciones. Finalmente, las proyecciones que esto tendría en el marco de la nueva reforma educacional comenzada en el 2014 por el gobierno de Michelle Bachelet, y donde debiese proyectarse una intervención curricular para incluir el tema antártico. Esto, por supuesto, desde la mirada de los datos que emergieron en esta investigación.

El análisis de los datos, es decir, de las referencias de los sujetos, permitió enmarcar las percepciones en seis categorías claves para su análisis; Historia y Conquista, Soberanía y Territorio, Medio ambiente y Desarrollo Científico, Utilidad Económica y finalmente, Abordaje y Limitaciones del Currículum.

En estas categorías fue posible identificar un trato de la temática desde dos miradas transversales; desde la mirada de lo Objetivo, es decir, desde la óptica de las instituciones pre existentes y la percepción que se tiene de ello en la experiencia de los sujetos entrevistados (Berger \& Luckmann, 1986) y desde la óptica del currículum prescrito, que ha sido tratado y expresado como los planes y programas formales de la asignatura de Historia, Geografía y Ciencias Sociales. Por otro lado, la acción subjetiva que los docentes han incorporado en el tratamiento de la temática al no estar presente en el currículum objetivo y desde la propia mirada en que esto se aborda por parte de los docentes.

En el primer punto, desde la mirada objetiva, es necesario desprender dos ejes claves; la pre existencia de las instituciones que dan origen a ciertos elementos analizados en esta investigación, como lo es el Tratado Antártico y la red de nuevas formas de comunicación a nivel nacional donde tal tratado se inserta tras la Segunda Guerra Mundial. Y es que, en este sentido, una de los significados más relevantes desprendidos de esta investigación radicaría en el no cuestionamiento a tal realidad institucional, la que además se proyectaría a cómo se percibe la Antártica mediante dicho Tratado, donde dada pre existente a la experiencia de los sujetos, la forma en que emerge diplomáticamente la Antártica, su defensa y preservación no sería un tema en construcción de la realidad -social- cotidiana, sino que al contrario, es inamovible.

Las características objetivas de esto, además se representan en que este tipo de Tratados, poco frecuentes y cuasi únicos en la historia, no alteraría dinámicas de comportamiento a nivel mundial, no implicarían riesgos tales como amenazas al orden establecido, y tampoco incidiría en el progreso económico de las naciones que ahí participan, lo que haría a tal pre existencia a la experiencia de los sujetos más lejana a la forma en que los países se comportan en la actualidad. De cierta manera, la realidad objetiva del periodo de Globalización, en el que se insertan mayoritariamente las experiencias de los entrevistados, no pretende o no es frecuente una red de relaciones que no vaya en pro de la cooperación económica entre las naciones.

Es en ese sentido, la Antártica, desde la forma en que ésta emerge en la historia mundial, poco representativa de las interconexiones mundiales que se han ido dando desde la segunda mitad del siglo XX en el mundo y además, el hecho de ser un escenario poco dinámico en su tratamiento diplomático, dejarían aún más encerrado el carácter institucional de ella. 
Desde la mirada de la Sociología del Conocimiento, no presentaría elementos de potencial incidencia en la construcción de la realidad cotidiana de los docentes al ser esto imposible de reconstruir sistemáticamente.

Otro elemento objetivo identificado en los datos analizados corresponde al currículum nacional en su carácter prescrito, donde es posible apreciar una valoración ciertamente positiva en las percepciones de los docentes. La idea de la obligatoriedad de los contenidos de los planes y programas de la asignatura permitiría que los docentes entrevistados expongan o no la temática en su clases, aún si el tema tuviera relevancia en la actualidad. Esto es posible desprenderlo en el sentido de que, si bien existió un consenso de la importancia de la temática para nuestra tricontinentalidad, el abordaje de ella aparentemente no es urgente al no estar contenido en el currículum prescrito.

En función de lo anterior, es necesario reconocer que dentro de estos elementos objetivos, existe una dimensión subjetiva propia de los docentes, en lo que respecta el abordaje de la temática y desde donde, en ciertas oportunidades y de acuerdo a sus propios intereses, posicionan la temática. Estas iniciativas, de abordar la temática comienzan y terminan en cada docente entrevistados al ser parte de iniciativas particulares, no colaborativas y desde el punto en que cada sujeto consideró pertinente abordar el tema.

Así es como encontramos dos manifestaciones de tal dimensión subjetiva; por un lado contenido en el concepto "Soberanía" y "Territorio" y que dada la forma en que se construyó esta investigación, también involucra a una categoría completa de análisis de los datos. En esta línea, los conceptos mencionados se escapan ampliamente de cómo estarían referenciados en la literatura científica e incluso desde la mirada Constitucional de nuestro pais, lo que además toma mayor relevancia cuando identificamos que es desde esta otra mirada, divergente a la teoría conocida, como identifican la Antártica y el territorio antártico chileno.

Por consiguiente, la soberanía nacional tomaría un carácter eminentemente territorial, en un sentido geográfico y físico y no desde el ejercicio de derechos, como está entendida en la Constitución. La importancia de lo anterior radica en que las percepciones de los docentes traspasan la esfera de los lineamientos conceptuales de nuestra propia institucionalidad política, corresponde a una visión subjetiva en relación a lo objetivo de la teoría institucional conteniendo elementos particulares a la realidad subjetiva de los docentes, lo que además es traspasado hacia el estudiantado al abordar la temática. Acción que por lo demás, también corresponde a la esfera lo subjetivo al no estar dentro del curriculum objetivo.

Finalmente, los docentes significan el territorio antártico desde una mirada ajena al currículum, respetando el carácter obligatorio de éste y considerando que si bien la temática no se encuentra presente en el, es relevante hablar de ella desde, en este caso, dimensiones altamente vinculadas al espacio físico y geográfico entendiendo esto como parte del ejercicio de la soberanía nacional, a pesar de que los lineamiento teóricos de tal quehacer institucional por parte del Estado no sea el que perciben los docentes. Es una máxima representación del 
espacio subjetivo en que está enmarcado el tema para los sujetos entrevistados, además de reafirmar el carácter fenomenológico de esta investigación.

Como segundo eje, es necesario analizar las percepciones que los docentes poseen del mismo currículum prescrito y nacional y cómo desde la Historia, la Geografía y/o las Ciencias Sociales se asocia o vincula a los temas abordados en esta investigación. En ese sentido, los docentes significan el territorio antártico chileno desde las tres dimensiones en que se desarrolla la disciplina, encontrándose vinculaciones de la temática además con otros importantes procesos históricos de nuestro país.

En ese sentido y específicamente en el área de Historia, la temática antártica se encuentra vinculada a los inicios de la historia de nuestro país, específicamente desde el período de Descubrimiento y Conquista del territorio, donde parte de las percepciones de los docentes estarían fundamentadas en los documentos legales que otorgaría a los conquistadores tomar el territorio (con toda le extensión que esto implicaría) y desde ahí, asociarlo a nuestro propio pasado-legado español. Ciertamente esta mirada permite entender la valoración que los docentes realizan al proceso de Conquista al que el país se encuentra asociado y vinculando a la Antártica, un territorio no conocido en aquella época también como parte de aquel proceso.

A la hora de analizar la construcción del Estado-Nación tras la Independencia y a lo largo del siglo XIX, donde además se viven las mayores transformaciones territoriales del país y donde además, el currículum releva aquellos procesos que implicaron grandes aportes económicos al país. En esa línea, el conflicto de Chile contra Perú y Bolivia en la llamada Guerra del Pacífico o del Salitre (1879-1884) es ampliamente considerado por los docentes entrevistados, esto ya que evidentemente y dada la significancia que poseen sobre la obligatoriedad del currículum, es necesario abordarlo como contenido obligado en el la asignatura.

Los docentes además perciben como relevantes los hechos célebres del siglo XX en la temática antártica chilena, en especial aquellos que hacen mención a la llamada "soberanía", entendida claro, desde las dimensiones físicas y geográficas en sus percepciones y cómo esto construye la historia de una parte importante de la característica tricontinental del país. Esta idea de soberanía emana esencialmente desde las acciones fundamentes de los gobiernos de turno, vale decir, Pedro Aguirre Cerda y Gabriel González Videla al marcar además hitos históricos cada uno en la historia antártica, no así los otros hechos que también podrían ser considerados como acto de soberanía, y que corresponderían por ejemplo al rescate realizado por la armada chilena en 1906 a cargo del piloto segundo Luis Pardo Villalón, un acto vinculado a instancias de gobierno pero fuera del alcance de los significados de los docentes en torno a la idea de soberanía.

Desde la Geografía, las percepciones de los docentes entrevistados se relacionarían en primera instancia a la dimensión histórica, entendiendo que el poder español que da origen a nuestra historia, proyectaba el nuevo territorio hasta los confines de lo que hoy conocemos como Antartandes, es por consiguiente una percepción asociada y deductiva de los macro procesos históricos de nuestra legislación original. 
Sin embargo, existe un consenso en que muy bien la temática antártica podría vincularse o estudiarse desde la mirada geográfica, pero ésta se encuentra en retirada del currículum formal, situación que además es reconocida por los docentes entrevistados, por lo que no podría ser una opción a la hora de intervenir el currículum, considerando además la aceptación y validación que se posee sobre el carácter prescrito de éste.

Desde las Ciencias Sociales, el tema pareciera insertarse de mejor manera en nuestros planes y programas nacionales, en el sentido de que hay una convergencia en la percepción de los usos actuales que se le da al territorio antártico y a la Antártica como tal. Lo anterior se entiende representando en temas vinculados además, a la contemporaneidad de los procesos o a los hechos acaecidos durante el siglo XX en el mundo. El Tratado Antártico, por lo tanto, se encuentra perfectamente establecido como una muestra gráfica y latente del cuidado que se pretende en áreas tales como: la diplomacia, la ciencia y el cuidado del medio ambiente, grandes tópicos que se han desarrollado a partir de la segunda mitad del siglo XX en nuestro planeta. Hablamos de Ciencias Sociales entonces, porque dada la importancia de tales tópicos, podemos estudiarlo desde diversas áreas del conocimiento y no necesariamente en Historia o Geografía.

Desde esa perspectiva, se concluye que efectivamente se hace necesario incluir el contenido antártico en los planes y programas de la asignatura, ya que permitiría entregar otras herramientas en el currículum que permitan entender y aprehender lo necesario para conocer y proteger el territorio y su sentido, en pro de continuar con un sistema preservado para la paz, la ciencia en el futuro.

En línea con lo apuntado en el párrafo precedente y con el tercer eje que plateamos, el contexto más adecuado donde podría insertarse la temática, de acuerdo a las percepciones de los docentes, radicaría en este último punto relacionado a los usos autorizados en la actualidad para la Antártica, es decir, desde el estudio asociado a la ciencia y la preservación de la paz.

A partir de las escasas o muy particulares referencias asociadas a temáticas tales como Historia y Conquista o Utilidad Económica, se puede desprender la idea de que la temática antártica no presenta gran relevancia en tales sentidos, es decir, desde la mirada histórica, específicamente desde el pasado originario español y los fundamentos legales que en el periodo emanaron, donde la pertinencia estaría más asociada a los hechos nacionales del siglo XX y por otro lado, desde las posibles utilidades económicas que pudieran extraerse desde el continente blanco. Esta última, sin embargo, a pesar de no ser significante en el sentido del estudio de las percepciones a nivel general, si es recurrente en uno de los sujetos lo que podría estudiarse más allá del análisis grupal y podría responder a las experiencias particulares del sujeto, no así del grupo mismo.

La pertinencia del tema, en relación a la recurrencia y al sentido común de las percepciones radicaría en el estudio desde el siglo XX sobre la temática Antártica, en las que desde la mirada nacional puede vincularse a la dimensión histórica, como parte del contenido de Historia de Chile y desde una perspectiva global a unidades vinculadas a la 
Historia Contemporánea o a las Ciencias Sociales, lo que incluso podría abrir más el desarrollo de la temática a otras disciplinas.

De acuerdo al currículum que los docentes utilizaron en su experiencia para aportar a los datos de esta investigación, en la que además es posible concluir que para los sujetos la temática antártica es relevante pero no imprescindible, este puede ser tratado como un elemento complementario a las dos áreas antes mencionadas; Historia de Chile, siglo XX e Historia Contemporánea y temas de Ciencias Sociales.

En la primera área, se propone intervenir la Unidad 1: "Transformación del rol del Estado y modernización de la sociedad en la primera mitad del siglo XX" de tercero medio, específicamente al hablar del período de los Gobiernos Radicales donde los contenidos específicos corresponderían a: Fijación de los límites del territorio antártico chileno en el gobierno de Pedro Aguirre Cerda, $1^{\circ}$ visita de un mandatario a la Antártica y suscripción al Tratado Antártico durante el gobierno de Gabriel González Videla.

Ahora bien, es necesario mencionar que si bien el Marco Curricular de los niveles de $3^{\circ}$ y $4^{\circ}$ se ha mantenido a pesar de la reforma educacional en curso, ya que la propuesta de las nuevas Bases Curriculares para estos niveles fueron rechazadas durante el 2017, por lo que al menos hasta la fecha, se continúan aplicando las actualizaciones del 2009 al currículum vigente, lo que se ha mantenido sin modificaciones.

Sin embargo, las nuevas Bases Curriculares para $1^{\circ}$ y $2^{\circ}$ medio propios de la reforma del 2014 se encuentran en su primer año de aplicación durante el 2018, por lo que en este contexto, la propuesta antes señalada debería trasladarse al nivel de $2^{\circ}$ medio en la Unidad 2 : "El fortalecimiento del Estado y la democratización social en el Chile de la primera mitad del siglo XX" dada la coherencia cronológica y temática entre los dos niveles expuestos.

Situación muy similar ocurre con la segunda área donde podría insertarse la temática antártica respecto a Historia Contemporánea y Ciencias Sociales, ya que de acuerdo al currículum aún vigente de $4^{\circ}$ medio, esto concuerda con la Unidad 3 "Los desafíos de insertarse en un mundo globalizado: Chile y sus regiones frente a la economía y los problemas globales” y que en las nuevas Bases Curriculares también concordaría con el nivel de $2^{\circ}$ medio en la Unidad 2: "De un mundo bipolar a un mundo globalizado: la Guerra Fría”. En ambas Unidades puede insertarse el Tratado Antártico como nuevo sistema de diplomacia que busca, a diferencia de otros Tratados afines, la no innovación en la Antártica a fin de transformarlo en el único continente del mundo en que debe prevalecer la paz y el cuidado del medio y en el Chile juega un rol muy importante.

Por lo tanto, a modo de proyección para los desafíos del nuevo currículum de la asignatura y de acuerdo a las percepciones de los docentes partícipes de esta investigación, la temática debiera estar incluida en el nivel de $2^{\circ}$ medio correspondiente a las Bases Curriculares que se están implementando desde el 2018.

Esto, ya que en este nivel se pretende y de manera muy oportuna para los fines de esta investigación a nivel curricular, vincular las dos grandes dimensiones en que los docentes 
perciben mayormente la relevancia de tratar la temática antártica y desde ahí, la defensa de nuestra tricontinentalidad. En otras palabras, desde cómo Chile en su tarea de fortalecer el rol del Estado durante la primera mitad del siglo XX logra insertarse en un sistema pacífico de preservación de un territorio específico a nivel planetario, única instancia conocida en la historia de la humanidad donde lo más importante no es el progreso económico, sino la paz y la ciencia, una nueva red de comunicación global que nos permitirá construir nuevos conocimientos y un futuro sin límites ni fronteras. Una oportunidad que sin duda, no podemos permitir que se nos diluya en la indiferencia y el tiempo.

\section{REFERENCIAS}

Alvarado, M. (2013). Nivelación de competencias y temas antárticos. Programa Diplomado Asuntos Antárticos, Punta Arenas, Universidad de Magallanes. Ms.

Aigneren, M. (2002). La técnica de recolección de información mediante los grupos focales. Revista Electrónica La sociología en sus escenarios, 7(20), 1-32.

Agulló, E. (1998). La centralidad del trabajo en el proceso de construcción de la identidad de los jóvenes: una aproximación psicosocial. Psicothema, 10(1), 153-165.

Andechaga, M. (octubre de 2015). Mi ciudad y la Antártida llegan a la escuela, educación ambiental y las TIC en la formación docente y en el aula. IV Jornadas de Enseñanza e Investigación Educativa en el campo de las Ciencias Exactas y Naturales. Congreso celebrado en Ensenada, Argentina. Recuperado de: https://bit.ly/2OXKQZB

Berger, P., \& Luckmann, T. (1986) La construcción social de la realidad., Buenos Aires: Amorrortu.

Casanova, M. (1998). La evaluación educativa, escuela básica. Madrid: Biblioteca del Normalista. Canales, M. (2006). Metodologías de la investigación social. Santiago de Chile: LOM Ediciones. Elliot, J., Sanger, J., Wood, M., Hull, C., \& Barret, G. (1986). Investigación/acción en el aula. Valencia: Generalitat Valenciana.

Ferrer, G., Valverde, G., \& Esquivel, J. (1999). Aspectos del curriculum prescrito en América Latina: Revisión de tendencias contemporáneas en curriculum, indicadores de logro, estándares y otros instrumentos. Programa de Promoción de la Reforma Educativa en América Latina y el Caribe: Grupo de Análisis para el Desarrollo.

Recuperado de: http://repositorio.minedu.gob.pe/handle/123456789/217

Gaete, M. González, V., \& Leiva, F. (2007). Propuesta de inclusión de contenidos históricoculturales al Programa de Primer Año Medio de los establecimientos educacionales de la región de Magallanes y Antártica Chilena, asociados a la promoción de la Identidad Regional. Tesis de grado para optar al grado de Licenciatura en Educación. Universidad de Magallanes, Punta Arenas.

Galeano, M. (2004). Estrategias de investigación social cualitativa: el giro en la mirada. Medellín: La Carreta. 
García, E., Rodríguez, G., \& Jil, J. (1996). Metodología de la Investigación Cualitativa. Málaga: Editorial Aljibe.

García, A. (2009). Textos escolares: Las Malvinas y la Antártida para la 'Nueva Argentina'. Antíteses, 2(4), 1033-1058.

Glatthorn, A. (1994). Constructivism: Implications for curriculum. International Journal of Educational Reform, 3(4), 449-455.

Gorostegui, J., \& Waghorn, R. (2012). Chile en la Antártica; nuevos desafíos y perspectivas. Estudio enmarcado en proyecto de tesis para optar al grado de Magíster en Política Exterior. USACH.

Mayanz, J. (2014). Aproximaciones sobre las invasiones biológicas en la Antártica para estudiantes de segundo ciclo básico ¿Cómo conservar el Continente Blanco?. Programa Diplomado en asuntos Antárticos, (manuscrito no publicado), Punta Arenas, Universidad de Magallanes

Martínez, V. (2013). Paradigmas de investigación Manual multimedia para el desarrollo de trabajos de investigación. Una visión desde la epistemología dialéctico crítica. Recuperado de: https://bit.ly/2Bwsr0j

Ministerio de Educación (2011). Programa de Estudio Primer Año Medio, Historia, Geografía y Ciencias Sociales. Chile: Ministerio de Educación. Recuperado de;

https://goo.gl/qQLaFJ

Merton, R. (1965). Teoría y estructura sociales. Fondo de Cultura Económica: México.

McFadyen, E. (2011). Antarctic Future-Education Learning about Antarctica in the 21st Century. A review of selected education resources available to primary teachers. University of Canterbury. Recuperado de: https://bit.ly/2TEBq8L

Palacios, C. (2016). Chile y el reclamo de la soberanía sobre el territorio Antártico (manuscrito no publicado), Programa Diplomado en Asuntos Antárticos, Punta Arenas, Universidad de Magallanes.

Panichini, A. (2016). Relación histórica entre el Continente Blanco y nuestro país y el rol de la asignatura de Historia en la enseñanza de contenidos Antárticos. Programa Diplomado en Asuntos Antárticos, Punta Arenas, Universidad de Magallanes.

Poves, M. (2005). Entrevista a Leire Pajín, Secretaria de Estado de Cooperación Internacional. Revista de Relaciones Internacionales, Economía, Defensa y Tecnología, 12, 24-27.

Romero, C. (2005). Categorización un aspecto crucial en la Investigación Cualitativa. Revista de Investigaciones Cesmag, 11(11), 113-118. 